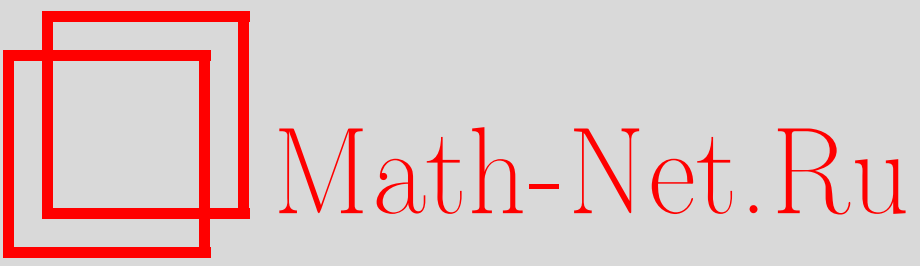

Л. Д. Пустыльников, Об асимптотическом поведении коэффициентов ряда Тейлора функции $\xi(s)$, УМH, 2000, том 55, выпуск 2, 145-146

DOI: https://doi.org/10.4213/rm280

Использование Общероссийского математического портала Math-Net.Ru подразумевает, что вы прочитали и согласны с пользовательским соглашением

http://www. mathnet.ru/rus/agreement

Параметры загрузки:

IP : 34.229 .108 .108

26 апреля 2023 г., 15:17:24 


\section{ОБ АСИМПТОТИЧЕСКОМ ПОВЕДЕНИИ КОЭФФИЦИЕНТОВ РЯДА ТЕЙЛОРА ФУНКЦИИ $\xi(s)$}

\section{Л. Д. Пустыльников}

В этой работе мы находим асимптотическую формулу для коэффициентов ряда Тейлора функции $\xi(s)=\frac{1}{2} s(s-1) \pi^{-s / 2} \Gamma(s / 2) \zeta(s)$ в точке $s=1 / 2$. Здесь $\Gamma(s)$ и $\zeta(s)$-соответственно гаммаи дзета-функции, а функция $\xi(s)$ является целой и в силу известного равенства $\xi(s)=\xi(1-s)$ разлагается в точке $s=1 / 2$ в ряд Тейлора

$$
\xi(s)=\sum_{r=0}^{\infty} \xi_{r}(s-1 / 2)^{2 r}
$$

только по четным степеням переменной $z=s-1 / 2$. Нахождение явного асимптотического выражения для коэффициентов $\xi_{r}$ ряда Тейлора функции $\xi(s)$ при $r \rightarrow \infty$ представляет интерес как самостоятельно, так и в связи с гипотезой Римана о нулях функции $\zeta(s)[1]$.

Теорема 1. При $r \rightarrow \infty$ справедливо асимптотическое равенство

$$
\begin{aligned}
\xi_{r} \sim \frac{2^{-(2 r-2)}}{(2 r) !}\left(\ln \frac{2 r-2}{\pi}-\ln \ln \frac{2 r-2}{\pi}+\beta\right)^{2 r-2} \exp \left(-(2 r-2)\left(\ln \frac{2 r-2}{\pi}\right)^{-1} e^{\beta}\right) \\
\quad \times(2 r-2)^{1 / 4}(2 r)(2 r-1)\left(\ln \frac{2 r-2}{\pi}\right)^{-1 / 4} \\
\quad \times \frac{\pi^{1 / 4}}{\sqrt{(r-1)\left(\frac{1}{\left(\ln \frac{2 r-2}{\pi}-\ln \ln \frac{2 r-2}{\pi}\right)^{2}}+\frac{1}{\ln \frac{2 r-2}{\pi}}\right)}},
\end{aligned}
$$

где $\beta=\beta(r)-$ такая функция, что $\lim _{r \rightarrow \infty} \beta(r)=0$.

Далее теорема 1 будет выведена из следующей теоремы 2.

Теорема 2. Пусть $r \geqslant 2, r-$ челое число, $\omega(x)=\sum_{n=1}^{\infty} \exp \left(-\pi n^{2} x\right)$,

$$
\mathrm{I}_{r}=\int_{1}^{\infty}\left(16 r(r-1)-\ln ^{2} x\right)\left(\ln ^{r-2} x\right) x^{-3 / 4} \omega(x) d x .
$$

Тогда при $r \rightarrow \infty$ справедливо асимптотическое равенство

$$
\begin{aligned}
\mathrm{I} r \sim 16 & \left(\ln \frac{r-2}{\pi}-\ln \ln \frac{r-2}{\pi}+\gamma(r)\right)^{r-2} \exp \left(-(r-2)\left(\ln \frac{r-2}{\pi}\right)^{-1} e^{\gamma}\right) \\
& \times(r-2)^{1 / 4} r(r-1)\left(\ln \frac{r-2}{\pi}\right)^{-1 / 4} \\
& \times \frac{\pi^{1 / 4}}{\sqrt{(r-2)\left(\frac{1}{2\left(\ln \frac{r-2}{\pi}-\ln \ln \frac{r-2}{\pi}\right)^{2}}+\frac{1}{2 \ln \frac{r-2}{\pi}}\right)}},
\end{aligned}
$$

әде $\gamma(r)-$ такая функция, что $\lim _{r \rightarrow \infty} \gamma(r)=0$.

Работа выполнена при частичной поддержке Российского фонда фундаментальных исследований (гранты № № 00-01-00583, 99-01-01063). 
ДокАЗАТЕЛЬСТво теОРемы 1. Мы воспользуемся известным равенством (см. [2], [3])

$$
\pi^{-\frac{s}{2}} \Gamma\left(\frac{s}{2}\right) \zeta(s)=\frac{1}{s(s-1)}+f(s)+g(s),
$$

где $s \neq 0,1$;

$$
\begin{aligned}
& f(s)=\int_{1}^{\infty} x^{\frac{s}{2}-1} \omega(x) d x \\
& g(s)=\int_{1}^{\infty} x^{-\frac{s}{2}-\frac{1}{2}} \omega(x) d x
\end{aligned}
$$

$\omega(x)$ - функция, введенная в формулировке теоремы 2.

Так как при любом натуральном числе $r$

$$
\frac{d^{r} f}{d s^{r}}=\left(\frac{1}{2}\right)^{r} \int_{1}^{\infty}\left(\ln ^{r} x\right) x^{\frac{s}{2}-1} \omega(x) d x
$$

и справедливо равенство $f(s)=g(1-s)$, то для любого четного числа $m$ имеем:

(3) $\frac{1}{2}\left(\frac{d^{m} f}{d s^{m}}\left(\frac{1}{2}\right)+\frac{d^{m} g}{d s^{m}}\left(\frac{1}{2}\right)\right)=\frac{d^{m} f}{d s^{m}}\left(\frac{1}{2}\right)=\left(\frac{1}{2}\right)^{m} \int_{1}^{\infty}\left(\ln ^{m} x\right) x^{-\frac{3}{4}} \omega(x) d x$.

Далее, представляя число $s$ в виде $s=1 / 2+z$, заметим, что $s(s-1)=z^{2}-1 / 4$. Поэтому, умножая обе части равенства (2) на величину $\frac{1}{2} s(s-1)$, в силу определения функции $\xi(s)$ и равенств (2), (3) получим для четной производной $\frac{d^{m} \xi}{d s^{m}}\left(\frac{1}{2}\right)$ следующее выражение:

$$
\frac{d^{m} \xi}{d s^{m}}\left(\frac{1}{2}\right)=m(m-1) \frac{d^{m-2} f}{d s^{m-2}}\left(\frac{1}{2}\right)-\frac{1}{4} \frac{d^{m} f}{d s^{m}}\left(\frac{1}{2}\right)=2^{-(m+2)} \mathrm{I}_{m}
$$

где $\mathrm{I}_{m}$ - величина, введенная в теореме 2 при $m=r$. Теперь утверждение теоремы 1 непосредственно следует из равенства (4) и теоремы 2 . Теорема 1 доказана.

ЗАмечание. Формула (4) для четной производной функции $\xi(s)$ в точке $s=1 / 2$ впервые приведена в работе [1], где также доказано,что все эти производные строго положительные (теорема 1 и ее доказательство).

Теорема 1 используется для доказательства следующего результата.

Теорема 3. При любом натуральном числе $r$ определим величинь

$$
a_{r}=\frac{\xi_{r}}{\xi_{0}}, \quad \delta_{r}=\frac{\left(r ! a_{r}\right)^{\frac{r+1}{r}}}{(r+1) ! a_{r+1}},
$$

где $\xi_{r}-$ числа, введеннье в (1). Тогда имеет место равенство

$$
\lim _{r \rightarrow \infty} \delta_{r}=e .
$$

Теорема 3 и численные расчеты нескольких первых значений $\delta_{r}$ позволяют сделать следующий вывод.

ГиПотЕЗА. При всех $r \geqslant 1$ справедливо неравенство $\delta_{r}>1$.

\section{СПИСОК ЛИТЕРАТУРЫ}

[1] Пустыльников Л. Д. // УМН. 1999. Т. 54. № 1. С. 260-261. [2] Карацуба А. А. Основы теории чисел. М.: Наука, 1975. [3] Karatsuba A. А., Voronin S. M. The Riemann Zeta-Function. Berlin: de Gruyter, 1992. 